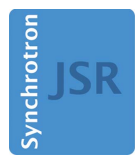

JOURNAL OF SYNCHROTRON RADIATION

ISSN 1600-5775

Received 13 March 2020

Accepted 20 July 2020

Edited by R. W. Strange, University of Essex, United Kingdom

Keywords: soft X-ray absorption; instrumentation; sum rules; magnetism; thin films.

\section{Soft X-ray absorption of thin films detected using substrate luminescence: a performance analysis}

\author{
Cinthia Piamonteze, ${ }^{\mathrm{a} *}$ Yoav William Windsor, ${ }^{\mathrm{a}, \mathrm{b}}$ Sridhar R. V. Avula, ${ }^{\mathrm{a}}$ \\ Eugenie Kirk $^{\mathrm{c}, \mathrm{d}}$ and Urs Staub ${ }^{\mathrm{a}}$

\begin{abstract}
${ }^{\mathbf{a}}$ Swiss Light Source, Paul Scherrer Institut, CH-5232 Villigen PSI, Switzerland, ${ }^{\mathbf{b}}$ Department of Physical Chemistry, Fritz Haber Institute of the Max Planck Society, Faradayweg 4-6, 14195 Berlin, Germany, 'Paul Scherrer Institut, 5232 Villigen PSI, Switzerland, and 'Laboratory for Mesoscopic Systems, Department of Materials, ETH Zurich, 8093 Zurich, Switzerland. *Correspondence e-mail: cinthia.piamonteze@psi.ch
\end{abstract}

X-ray absorption spectroscopy of thin films is central to a broad range of scientific fields, and is typically detected using indirect techniques. X-ray excited optical luminescence (XEOL) from the sample's substrate is one such detection method, in which the luminescence signal acts as an effective transmission measurement through the film. This detection method has several advantages that make it versatile compared with others, in particular for insulating samples or when a probing depth larger than $10 \mathrm{~nm}$ is required. In this work a systematic performance analysis of this method is presented with the aim of providing guidelines for its advantages and pitfalls, enabling a wider use of this method by the thin film community. The efficiency of XEOL is compared and quantified from a range of commonly used substrates. These measurements demonstrate the equivalence between XEOL and X-ray transmission measurements for thin films. Moreover, the applicability of XEOL to magnetic studies is shown by employing XMCD sum rules with XEOL-generated data. Lastly, it is demonstrated that above a certain thickness XEOL shows a saturation-like effect, which can be modelled and corrected for.

\section{Introduction}

The study of heterostructures and thin films of complex systems has become very important due to the novel properties that can be obtained at the interface of dissimilar materials or stabilized by strain engineering (Zubko et al., 2011). The element-specific information provided by soft X-ray absorption spectroscopy (XAS) combined with the magnetic and orbital information provided by X-ray magnetic circular dichroism (XMCD) and X-ray linear dichroism (XLD), respectively, made these techniques widely used in investigation of thin films.

Most materials of interest in this field contain $3 d$ transition metals or lanthanides, for which large XMCD and XLD contrast appears for $L_{2,3}(2 p \rightarrow 3 d$ transitions $)$ and $M_{4,5}(3 d \rightarrow$ $4 f$ transitions), respectively. These edges lie at energies where the X-ray mean free path is of the order of $50 \mathrm{~nm}$ to $100 \mathrm{~nm}$. This makes transmission measurements for epitaxially grown films and heterostructures challenging. Therefore, indirect detection methods like total electron yield (TEY) or total fluorescence yield (TFY) are commonly used. TEY is a measurement of the drain current from the sample, which is created to compensate for electrons ejected as a consequence of the X-ray absorption process. The TEY probing depth is typically $5-10 \mathrm{~nm}$ and requires a conducting surface. TFY measures fluorescence X-ray photons generated due to the photo-absorption effect, and therefore probes to depths of 
around $100 \mathrm{~nm}$. Moreover, since photons are measured, the samples do not need to be conducting. However, while spectra acquired from TEY are often used for sum rule analysis of XMCD data, this is not reliable for TFY, as it suffers from selfabsorption (Iida \& Noma, 1993; Tröger et al., 1992) and often it does not reflect the intrinsic XAS (de Groot, 2012). To overcome the limitations of TFY, the use of inverse partial fluorescence yield can be employed (Achkar et al., 2011). In this method one measures non-resonant $\mathrm{X}$-ray emission by using an energy-dispersive fluorescence detector.

$\mathrm{X}$-ray excited optical luminescence (XEOL) is an alternative detection method for obtaining XAS of thin films or heterostructures. X-rays absorbed in an insulator create highenergy electrons that produce further conduction electrons and valence holes by electron-electron scattering. Some of these electron-hole pairs radiatively recombine through defect states emitting luminescence (Elango, 1994). This gives rise to the so-called XEOL signal. This technique was initially proposed as a way to detect site-specific XAS in the hard X-ray energy range (Bianconi et al., 1978). In recent years $\mathrm{XEOL}$ has been introduced as an alternative detection method for measuring soft X-ray transmission through thin films (Kallmayer et al., 2007; Vaz et al., 2012, 2013). X-rays transmitted through the film create optical luminescence in the substrate. This method has been applied to collect XAS and XMCD from thin oxide films (Kallmayer et al., 2007; Meinert et al., 2011; Cao et al., 2015; Green et al., 2015; Windsor et al., 2017; Aeschlimann et al., 2018) and also for spatially resolved measurements using scanning transmission X-ray microscopy (STXM) (Vaz et al. 2012, 2013). Vaz et al. (2013) discuss in detail the origin of the photoluminescence in some substrates. For example, in $\mathrm{MgO}$ the photoluminescence is associated with $\mathrm{Cr}^{3+}$ impurities, while in LAO the photoluminescence is attributed to defects in twin boundaries.

In this work we explore the strengths and shortcomings of this detection method, which may allow its wider use in the research of strain engineered thin films and heterostructures. The strength of the XEOL method is that it probes the whole thin film thickness and allows measuring insulating films. For planning XAS measurements by XEOL, it is important to know the X-ray luminescence efficiency of the substrate. For this reason we present here the XEOL efficiency for different substrates. In addition we discuss the application of XMCD sum rules. Finally, we give guidelines for limits on film thickness required to avoid artefacts in the measurements and we propose a method to correct for saturation-like effects in the measurements.

\section{Experimental}

Co films of $20 \mathrm{~nm}$ thickness were DC-magnetron sputtered in one deposition on nine commonly employed substrates listed in Table 1. All substrates were $0.5 \mathrm{~mm}$ thick. For quantifying the thickness effects, Co films of 10, 40 and $80 \mathrm{~nm}$ thickness were sputtered on $\mathrm{MgO}$. Lastly, a reference sample was produced for measuring X-ray transmission: a $20 \mathrm{~nm}$-thick Co film was sputtered on $100 \mathrm{~nm}$-thick $\mathrm{Si}_{3} \mathrm{~N}_{4}$. All films were
Table 1

XEOL efficiency measured for bare substrates in grazing incidence. Measurements at $\left(^{*}\right) 300 \mathrm{~K}$ and $\left(^{* * *}\right) 100 \mathrm{~K}$.

\begin{tabular}{lll}
\hline Substrate & Abbreviation & XEOL efficiency \\
\hline $\mathrm{YAlO}_{3}$ & YAO & $2.7 \times 10^{-1 *}$ \\
$\mathrm{MgAl}_{2} \mathrm{O}_{4}$ & MAO & $1.1 \times 10^{-1 * *}$ \\
$\mathrm{Cr}: \mathrm{SrTiO}_{3}$ & Cr:STO & $4.5 \times 10^{-2 * *}$ \\
$\mathrm{MgO}$ & $\mathrm{MgO}$ & $2.8 \times 10^{-2} *$ \\
$\mathrm{SrTiO}_{3}$ & STO & $2.5 \times 10^{-2 * *}$ \\
$\mathrm{SrTiO}_{3}$ & STO & $8.7 \times 10^{-4 *}$ \\
$\left(\mathrm{LaAlO}_{3}\right)_{0.3}-\left(\mathrm{Sr}_{2} \mathrm{AlTaO}_{6}\right)_{0.7}$ & LSAT & $4.2 \times 10^{-3} *$ \\
$\mathrm{LaAlO}_{3}$ & LAO & $1.2 \times 10^{-2 *}$ \\
$\mathrm{NdGaO}_{3}$ & NGO & $1.0 \times 10^{-4 *}$ \\
$\mathrm{DyScO}_{3}$ & DSO & $6.1 \times 10^{-5 *}$ \\
\hline
\end{tabular}

capped with $3 \mathrm{~nm}$ or $5 \mathrm{~nm}$ sputtered $\mathrm{Pt}$ before breaking vacuum, to avoid oxidation. The film thicknesses were verified by X-ray reflectivity.

The X-ray absorption measurements were carried out at the X-Treme beamline (Piamonteze et al., 2012) at the Swiss Light Source. The XAS spectra were obtained by taking the natural logarithm of the normalized data, following the Beer-Lambert law for linear absorption,

$$
I=I_{0} \exp (-\mu t) \Rightarrow \mu t=\ln \left(I_{0} / I\right) .
$$

In the equation above, $I$ and $I_{0}$ are the transmitted and incident X-ray intensities, respectively. $\mu$ is the linear absorption coefficient and $t$ is the film thickness. In our case $I$ is the XEOL signal and $I_{0}$ is measured in a gold mesh upstream from the sample. To account for the energy dependence of XEOL, we normalize the transmission signal $\left(I^{\mathrm{T}}=I / I_{0}\right)$ measured from the Co/substrate by the transmission measured from the bare substrate. Finally we take the logarithm to obtain the actual absorption spectrum. The raw XEOL data for the films is presented in Section 3.1. The XEOL signal was measured using a UVG100 photodiode. The photodiode was mounted directly behind the sample, assuring a large solid angle collection. The incoming X-ray flux, used to create Table 1, was measured using an AXUV100 photodiode and its quantum efficiency was given by Kjornrattanawanich et al. (2006). Both photodiodes were designed by IRD and are sold by OptoDiode (http://optodiode.com).

XMCD measurements of the Co/substrate films were carried out with a field of $2 \mathrm{~T}$ applied parallel to the X-ray beam. The films were at $60^{\circ}$ incidence angle (between the film normal and the X-ray beam). The measurements were carried out at $300 \mathrm{~K}$ unless otherwise noted.

The data for $80 \mathrm{~nm} \mathrm{Co} / \mathrm{MgO}$ in $60^{\circ}$ incidence had spikes at the minimum transmission which were removed from the raw data for better visualization of the absorption spectrum.

\section{Results and discussion}

\subsection{Raw data}

Fig. 1(a) shows the XEOL data from the $\mathrm{Co} / \mathrm{MgO}$ thin films of various thickness and from bare $\mathrm{MgO}$ substrate. The $\mathrm{XEOL}$ signals for the thin films are divided by the bare substrate signal. Then the XAS are calculated by taking the natural 

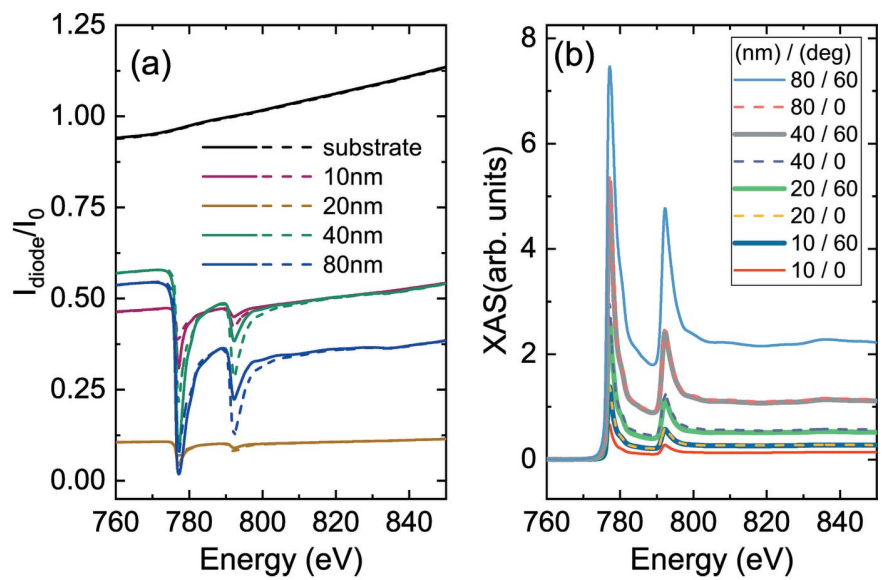

Figure 1

(a) $I_{\text {diode }} / I_{0}$ from $\mathrm{Co} / \mathrm{MgO}$ films with different Co thickness and bare $\mathrm{MgO}$ substrate. The continuous and dashed lines are the data for positive and negative helicity, respectively. (b) XAS for $\mathrm{Co} / \mathrm{MgO}$ with different $\mathrm{Co}$ thickness (nm) and different incidence angles (degrees).

logarithm of the normalized data. Fig. 1(b) shows the XAS data obtained after background subtraction.

In Fig. 1(a) it is clear that the $20 \mathrm{~nm}$-thick film has a different background than the others. This sample was prepared in a different batch and had a different thickness of Pt capping. However, as shown in Fig. 1(b), the edge jump scales well with the other samples. In grazing incidence $\left(=60^{\circ}\right.$ incidence) the effective thickness is twice the actual film thickness. Therefore, as expected, the grazing incidence measurement for each thickness overlaps with the normal incidence measurement of the film with double nominal thickness. For example, the spectrum measured for the nominal $80 \mathrm{~nm}$ film in normal incidence superposes the measurement from the $40 \mathrm{~nm}$ film in $60^{\circ}$ incidence, as seen in Fig. 1(b).

\subsection{Comparison with $X$-ray transmission}

We now demonstrate the equivalence of XEOL and transmission measurements. Figs. 2(a) and 2(b) present a direct comparison between the two measurements, which are sketched in Fig. 2(c). The figures present XAS and XMCD spectra measured at the Co $L_{3,2}$-edges from $20 \mathrm{~nm}$-thick Co films deposited in parallel on $\mathrm{Si}_{3} \mathrm{~N}_{4}$ and on $\mathrm{MgO}$. The $\mathrm{Co} / \mathrm{Si}_{3} \mathrm{~N}_{4}$ data were collected in transmission while the $\mathrm{Co} / \mathrm{MgO}$ data were collected using XEOL. The excellent agreement between them demonstrates the equivalence of these detection modes.

\subsection{XEOL efficiency}

We now discuss the efficiency of XEOL measurements for different substrates. We have calculated the values for XEOL efficiency by dividing the XEOL flux of the bare substrate by the incoming X-ray flux $\left(F_{\mathrm{XEOL}} / F_{\mathrm{X} \text { rays }}\right)$. In order to calculate the flux we used the expression

$$
F=\frac{I_{\text {diode }}}{q Q_{\text {eff }}}
$$

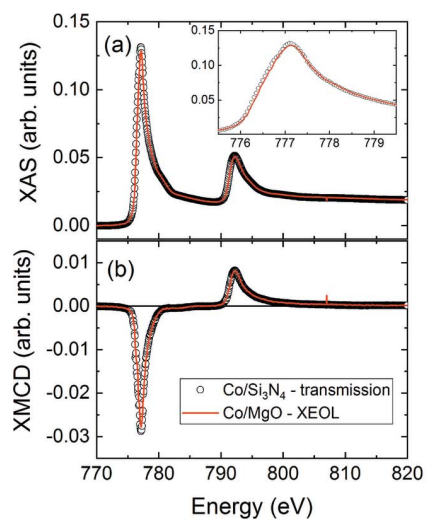

(c)

\section{Figure 2}

(a) XAS and (b) XMCD at the Co edge for $\mathrm{Co} / \mathrm{Si}_{3} \mathrm{~N}_{4}$ measured in transmission (open circles) and $\mathrm{Co} / \mathrm{MgO}$ measured using XEOL (line). The inset shows a magnification of the $L_{3}$-edge. (c) Sketch of the measurement setup for XEOL and transmission measurements.

where $F$ is the flux in photons per second, $I_{\text {diode }}$ is the current measured at the photodiode in Ampère, $q$ is the electron charge and $Q_{\text {eff }}$ is the quantum efficiency (Kjornrattanawanich et al., 2006).

The responsivity relates to the quantum efficiency by the following expression,

$$
R=\frac{Q_{\text {eff }} \lambda}{1.24}
$$

Therefore,

$$
\frac{F_{\mathrm{XEOL}}}{F_{\mathrm{Xrays}}}=\frac{I^{\mathrm{XEOL}} \lambda^{\mathrm{XEOL}}}{I^{\mathrm{Xrays}} \lambda^{\mathrm{Xrays}}} \frac{R^{\mathrm{Xrays}}}{R^{\mathrm{XEOL}}} .
$$

In the equation above, $R^{\text {Xrays }}$ stands for the responsivity of the photodiode measuring the $\mathrm{X}$-ray flux and $R^{\mathrm{XEOL}}$ is the responsivity of the photodiode used to measure the XEOL signal. $\lambda^{\mathrm{XEOL}}$ and $\lambda^{\mathrm{Xrays}}$ are the wavelength of the corresponding radiation incident on the photodiode. The responsivities of both photodiodes are provided by the supplier (http://optodiode.com). The responsivity used for the conversion of the photocurrent of the incoming X-rays was $0.25 \mathrm{~A} \mathrm{~W}^{-1}$, which is the responsivity at $760 \mathrm{eV}(1.6 \mathrm{~nm})$ for AXUV100. For the XEOL flux calculation the responsivity used was $0.48 \mathrm{~A} \mathrm{~W}^{-1}$, which is the responsivity for $1.94 \mathrm{eV}$ $(639.5 \mathrm{~nm})$ radiation, which is between the luminescence peaks measured by LAO and $\mathrm{MgO}$ (Vaz et al., 2013). The photodiode used measures an energy-integrated signal and its working wavelength range is between $300 \mathrm{~nm}$ and $1000 \mathrm{~nm}$ with optimal efficiency at $800 \mathrm{~nm}$.

Table 1 lists the XEOL efficiency and Fig. 3 presents the $\mathrm{XEOL}$ signal of the bare substrates as a function of X-ray energy near the Co $L$-edges. The XEOL efficiency reflects how many optical luminescence photons are produced per $\mathrm{X}$-ray photon that reaches the substrate. Notice that the photoluminescence is temperature dependent (Grabner, 1969). For STO, for example, the efficiency increases by almost a factor of 30 when cooling to $100 \mathrm{~K}$. Similarly, MAO and Cr:STO gave very weak XEOL signal at room temperature (RT) and only the efficiency at $100 \mathrm{~K}$ was measured. Even 


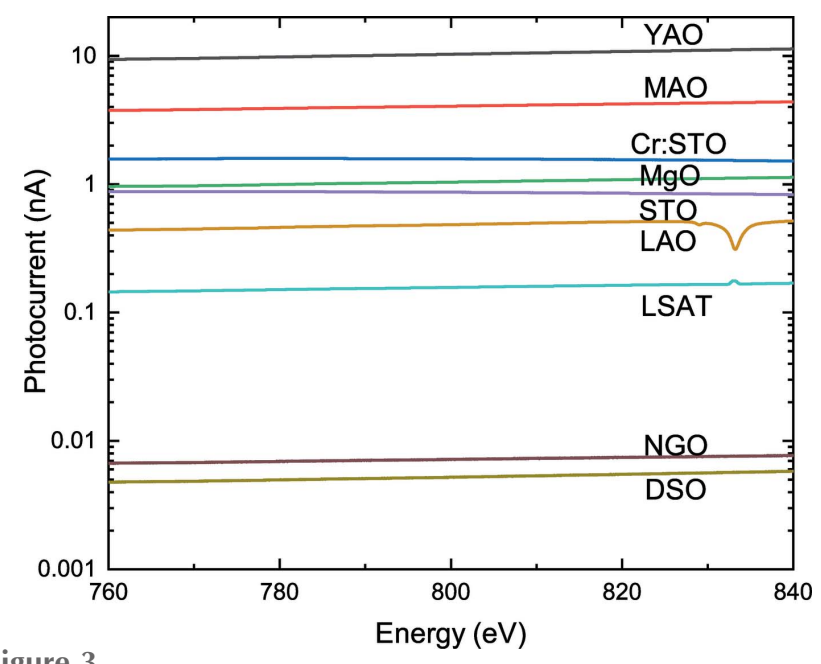

Figure 3

XEOL photocurrent measured behind the bare substrate at $60^{\circ}$ incidence. The measurements were made at room temperature, except for MAO, STO and Cr:STO which were measured at $100 \mathrm{~K}$. The normal flux conditions used for almost all substrates was with a front-end slit opening of $0.5 \mathrm{~mm} \times 0.5 \mathrm{~mm}$. For DSO and NGO the horizontal frontend slit was further open to $1.0 \mathrm{~mm}$ and $1.7 \mathrm{~mm}$, respectively.

though the XEOL signal of STO at RT is very weak, at $100 \mathrm{~K}$ it is comparable with $\mathrm{MgO}$ at room temperature. Moreover, the addition of $\mathrm{Cr}$ impurities increases the XEOL signal from STO by almost a factor of two at $100 \mathrm{~K}$. DSO and NGO substrates produce a very low XEOL signal, as shown in Table 1. For this reason the data shown in Fig. 3 for DSO and NGO were measured with approximately three and two times higher flux, respectively, than for the other substrates. Since the luminescence depends on impurities and crystal defects, there could be variations depending on the substrate manufacturer. Even so, Table 1 gives the order of magnitude of the expected signal when designing an experiment using XEOL as detection mode.

\subsection{Scanning speed}

In many contemporary soft X-ray beamlines, scanning of the monochromator is conducted continuously and not in discrete steps. If the luminescence decay is very slow, there could be an effect of the scanning speed on the spectrum. We have therefore checked the effect of scanning speed for all samples measured here. One example is shown in Fig. 4 for $20 \mathrm{~nm}$ Co deposited on $\mathrm{MgO}$ capped with $3 \mathrm{~nm}$ of $\mathrm{Pt}$, measured at two different scanning speeds. The difference between the two XAS spectra is also plotted. As seen from this figure, the difference between the spectra measured at different scanning speeds is of the order of $2.0 \%$ of the XAS maximum. This difference comes from a later rise of the edge for the faster scanning speed. The difference in energy between the inflection point of the two scans is about $25 \mathrm{meV}$.

The core hole life time broadening of $2 p$ core levels is often above $200 \mathrm{meV}$ (Krause \& Oliver, 1979). The narrowest feature measured at the transition metal $L$-edge of oxides is probably the $t_{2 g}$ resonance at the $L_{3}$-edge XAS of $\mathrm{Ti}^{4+}$, which has a lifetime broadening of $100 \mathrm{meV}$ (de Groot et al., 1990).

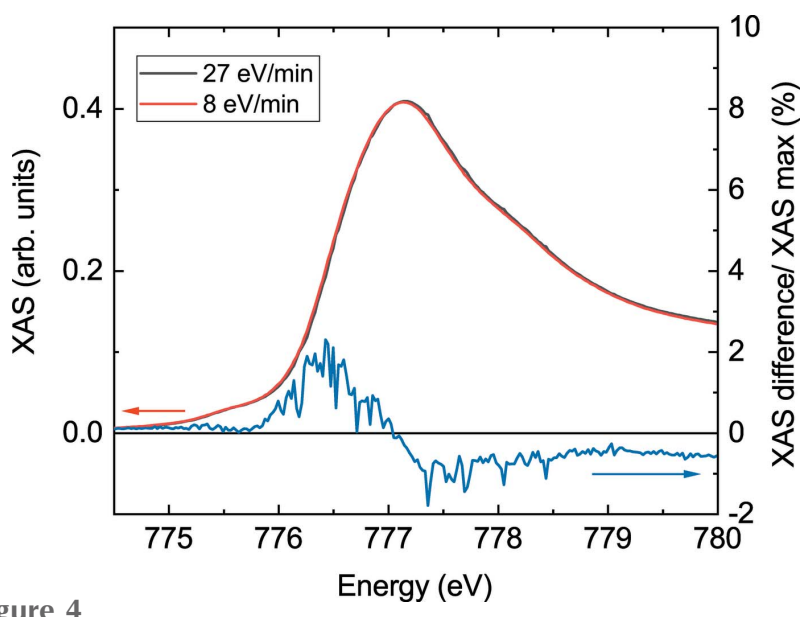

Figure 4

XAS at the Co $L_{3}$-edge measured at different scanning speeds of the monochromator. The blue curve corresponds to the difference between the two XAS spectra.

Therefore, the change in the structure width of $25 \mathrm{meV}$ is smaller than the lifetime broadenings intrinsic to soft X-ray absorption spectra, but in some cases it could create a sizeable distortion. Therefore, when measuring spectra with very sharp features using XEOL it is advisable to check whether the scanning speed has a significant effect on the spectrum shape.

\subsection{XMCD sum rules}

We now focus on the application of XMCD sum rules on data generated using XEOL. Sum rules were applied to XMCD spectra of Co deposited on different substrates in order to obtain $m_{1}$ and $m_{s_{-} \text {eff }}$ (Thole et al., 1992; Carra et al., 1993), where $m_{1}$ is the angular magnetic moment and $m_{s_{-} \text {eff }}$ is the effective spin moment. The resulting spin and orbital moments are presented in Figs. 5(a) and 5(b). Because photoluminescence can depend on the incident photon energy, we tested whether the results of the sum rules analysis are affected by normalizing the XEOL signal of thin film by the XEOL signal from bare substrates, which were measured in the same energy range. The filled symbols in Figs. 5(a) and $5(b)$ correspond to the data corrected by the bare substrate measurement as done by Kallmayer et al. (2007), except that we did not normalize at the pre-edge. The open symbols represent the analysis results without the bare substrate
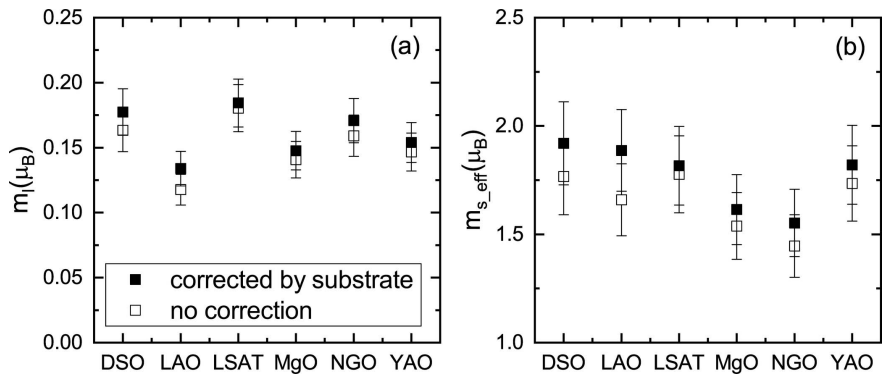

Figure 5

(a) Angular magnetic moment $m_{1}$ and (b) effective spin moment $m_{s_{-} \text {eff }}$ obtained from sum rule analysis of the films in different substrates. The error bars correspond to $\pm 10 \%$ of the moment value. 


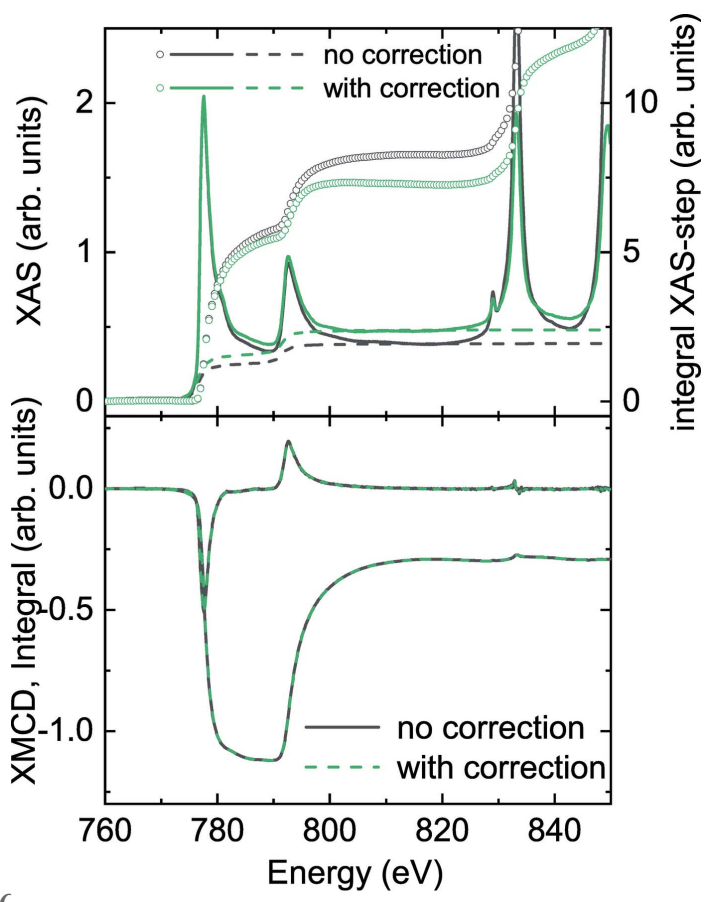

Figure 6

Mesurements for Co/LAO film. Top: XAS spectra (continuous line), step function (dashed line) and integral (circles) of XAS spectra after the subtraction of the step function. Bottom: XMCD and respective XMCD integral. In both panels the curves in black show the spectra where no substrate correction was made and in green are the curves where the XAS was corrected by the measurement of the bare substrate.

normalization. We find that both $m_{1}$ and $m_{s_{-} \text {eff }}$ are systematically reduced when the data are not normalized by the bare substrate data. The variations are on average below $10 \%$, which are the commonly used error bars for the XMCD sum rules (van der Laan, 1999). The exception is for Co/LAO where the substrate correction changes the sum rule result by $13 \%$. The effect of the substrate correction is to modify the slope of the XAS spectra and as a consequence it changes the XAS integral. This is illustrated in Fig. 6 where we show the XAS and XMCD integrals for the Co/LAO film. From this figure it also becomes clear why the substrate correction has such a large effect on the $\mathrm{Co} / \mathrm{LAO}$ measurement. The $\mathrm{La} M_{5,4^{-}}$ edges partially overlap with the Co absorption spectrum. In order to minimize the interference of the La spectra, the integral values for the sum rules were taken at $820 \mathrm{eV}$. The effect of the LAO substrate exemplifies an obvious problem for the use of XEOL detection: the energy range of interest should not significantly overlap with resonances of the substrate.

\subsection{Film thickness}

XEOL may be used to overcome the known shortcomings of other detection modes. TFY detection suffers from selfabsorption for samples with high concentrations of the absorbing element (Tröger et al., 1992; Iida \& Noma, 1993), while TEY detection exhibits saturation effects for very shallow incidence angles (Nakajima et al., 1999). For XEOL, there could also be self-absorption of the photoluminescence
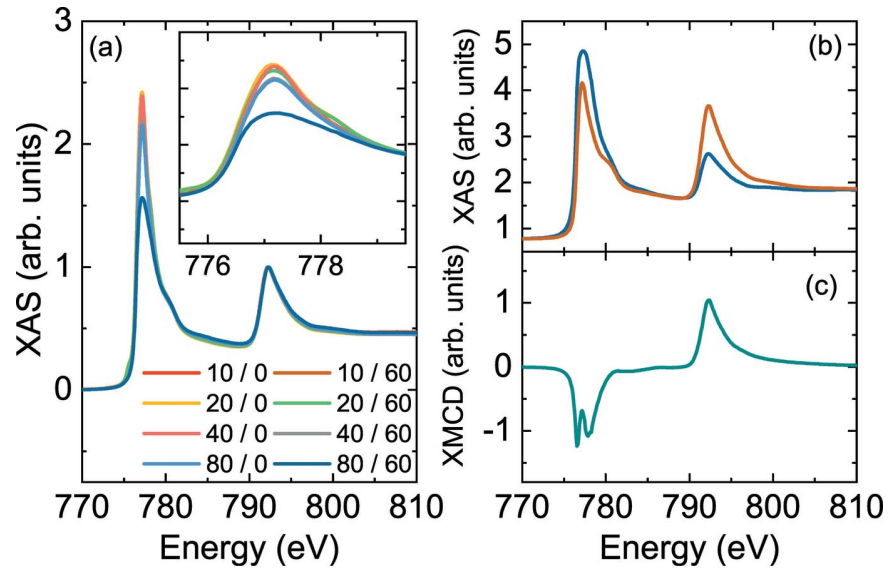

Figure 7

(a) XAS data normalized at the $L_{2}$-edge. The legend shows the nominal thickness in $\mathrm{nm} /$ incidence angle in degrees. (b) XAS for left (red) and right (blue) circular polarization and (c) XMCD for the $80 \mathrm{~nm}$-thick film at $60^{\circ}$ incidence.

at the substrate. However, the good agreement between XEOL and transmission in Fig. 2 indicates that this is not visible for $0.5 \mathrm{~mm}$-thick $\mathrm{MgO}$, probably due to the low absorption cross section of the optical photons. Transmission measurements should not saturate, but a saturation-like effect has been observed for samples with inhomogeneous thickness (Hanhan et al., 2009). Given that XEOL is a transmission equivalent measurement, we checked whether it suffers from the same saturation-like effects. We have measured Co films of several thicknesses at $0^{\circ}$ and $60^{\circ}$ incidence angles. The corresponding XAS spectra normalized at the maximum of the $L_{2}$-edge are presented in Fig. 7 (see Section 3.1 for the raw data). We find that the branching ratio between $L_{3}$ and $L_{2}$ depends on thickness, with larger thickness exhibiting a saturation-like effect at the $L_{3}$-edge. That is specially true for the $80 \mathrm{~nm}$-thick sample at $60^{\circ}$ incidence (i.e. effective thickness of about $160 \mathrm{~nm}$ ). Figs. 7(b) and 7(c) present the XAS and XMCD spectra for this film, where it can be seen that the measured $L_{3}$ XMCD appears clearly quenched.

Next we investigate how the saturation effect affects the sum rules results. Given that the orbital moment is proportional to the total XMCD integral, it is much more susceptible to the saturation effect than $m_{s_{-} \text {eff }}$. Figs. $8(a)$ and $8(b)$ present results of the sum rules analysis applied to the Co films of different thicknesses. Indeed, the effective spin moment does not vary much among the samples. The orbital moment clearly decreases as the thickness increases. For the $80 \mathrm{~nm}$ film at $60^{\circ}$ incidence, for which saturation is clearly seen in Figs. $7(b)$ and $7(c)$, the orbital moment even changes sign. In Section 3.8 we discuss how to correct this. Before that we discuss in Section 3.7 whether the X-ray flux has an effect on the saturation observed.

\subsection{Influence of X-ray flux}

In order to check whether the saturation observed was due to some fixed offset in the detection system we have measured all samples with different settings of the beamline where the 

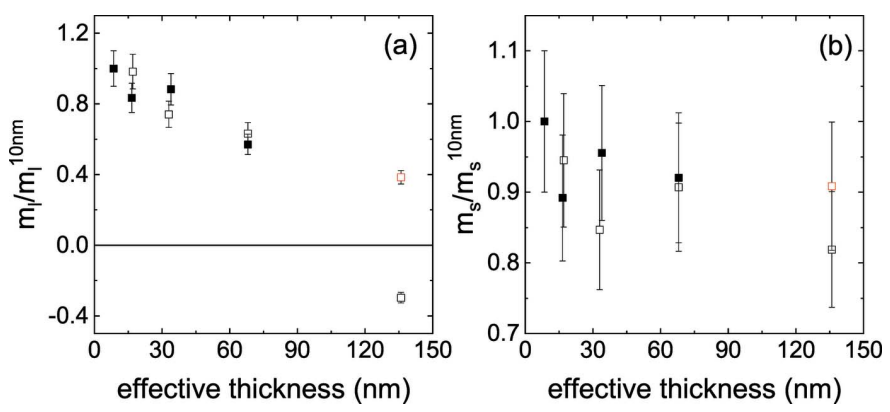

Figure 8

(a) Orbital moment and (b) effective spin moment plotted as a function of effective thickness. The data were normalized by the values for the $10 \mathrm{~nm}$ Co film in normal incidence. Filled symbols show the data measured in normal incidence while open symbols show the measurements at $60^{\circ}$ incidence. The red open square corresponds to the $80 \mathrm{~nm}$ data in grazing incidence after the saturation correction discussed in Section 3.8.

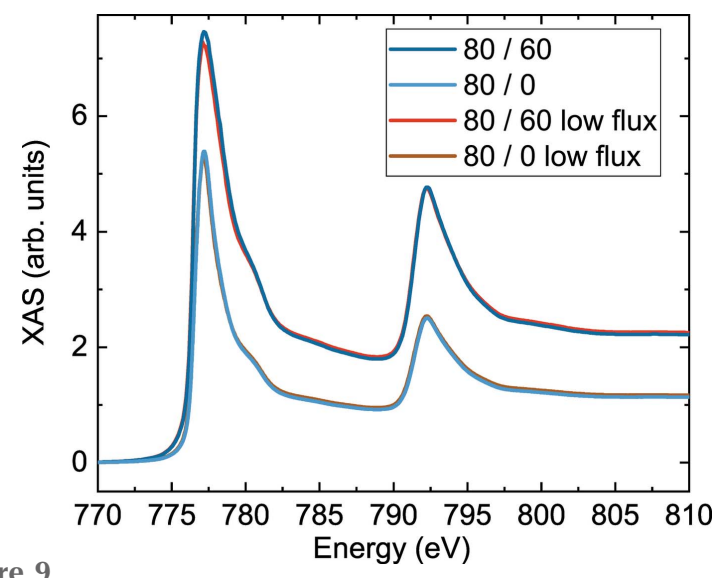

Figure 9

XAS for a nominal $80 \mathrm{~nm}$-thick sample measured in normal and grazing incidence compared with a measurement with one order of magnitude less flux.

flux was changed from about $2 \times 10^{11}$ photon $^{-1}$ to one order of magnitude less flux. If the saturation-like effect comes from an offset in the detection system, the spectra measured with ten times less flux should appear strongly saturated. Fig. 9 shows a comparison between high- and low-flux measurements for the $80 \mathrm{~nm}$ sample in normal- and grazing-incidence measurements. The XAS with high- and low-flux conditions are, within the precision of the measurement, identical to each other. This rules out that the observed saturation-like effect comes from an offset at the detector.

\subsection{Saturation correction}

We now model and correct the data for such saturation-like effects. As pointed out by Stern \& Kim (1981), hard X-ray transmission signals can exhibit a saturation effect if part of the incoming X-rays are not absorbed by the sample, for example due to high-order contamination in the incoming beam or pinholes on the sample. For $b(E)$ being the contribution to the incoming X-rays which is not absorbed by the sample, the measured normalized transmitted signal is given by

$$
\frac{I^{\prime}}{I_{0}^{\prime}}=\frac{I+b}{I_{0}+b}=\frac{I_{0} \exp (-\mu t)+b}{I_{0}+b} .
$$

In equation (5), $I_{0}^{\prime}$ and $I^{\prime}$ correspond to the measured quantities, while $I_{0}$ and $I$ represent the intrinsic quantities. If we call $\alpha(E)=b(E) / I_{0}(E)$, we obtain,

$$
\frac{I_{0}^{\prime}}{I^{\prime}}=\frac{1+\alpha}{\exp (-\mu t)+\alpha} .
$$

Equation (6) shows how the measured data relate to the linear absorption coefficient $\mu$ and to $\alpha$.

In Fig. 10(a) is plotted the ratio of the transmitted signals $I^{\mathrm{T}}=I / I_{0}$, taken at the energy of maximum absorption $\left(\sim 777.15 \mathrm{eV}\right.$, named $\left.I_{L 3}^{\mathrm{T}}\right)$ over those taken at the pre-edge $\left(\sim 760 \mathrm{eV}\right.$, named $\left.I_{\text {pre }}^{\mathrm{T}}\right)$. The curves in Fig. $10(a)$ are simulations based on expression (6) for two different values of the absorption coefficient $\mu$ at the resonance: $\mu=59 \mu \mathrm{m}^{-1}$ as obtained by Regan et al. (2001) and $\mu=45 \mu \mathrm{m}^{-1}$, which best describes our data. The change in $\mu$ only affects the agreement with the data in the low thickness range. An absorption coefficient of $2 \mu \mathrm{m}^{-1}$ was used for the pre-edge in all cases. For the black curves no saturation effect is considered $(\alpha=0)$. This results in a linear thickness dependence of the ratio $I_{L 3}^{\mathrm{T}} / I_{\text {pre }}^{\mathrm{T}}$, as expected. The red curves in Fig. 10(a) are for $\alpha=1.4 \%$. The simulation for $\alpha=1.4 \%$ and $\mu=45 \mu \mathrm{m}^{-1}$ is in good agreement with the measured data. The aim here is not to obtain a perfect agreement but to obtain an understanding for the saturation and attempt to correct for it. For simplicity, we consider $\alpha$ as a constant, but it could be energy dependent.

If now we invert expression (6) to obtain the intrinsic $I / I_{0}$ and consider $\alpha \ll 1$ we obtain

$$
\frac{I}{I_{0}}=\frac{I^{\prime}}{I_{0}^{\prime}}-\alpha
$$
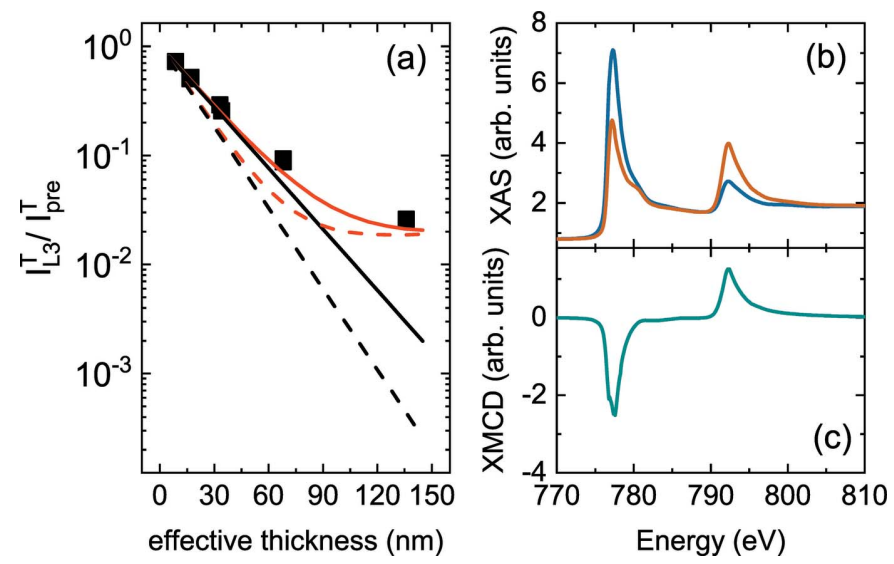

Figure 10

Transmitted intensity $\left(I^{\mathrm{T}}=I / I_{0}\right)$ taken at the peak of the $L_{3}$-edge divided by the $I^{\mathrm{T}}$ value at the pre-edge plotted against the effective thickness. Black (red) curves correspond to simulation for $\alpha=0(\alpha=1.4 \%)$. The continuous (dashed) curve corresponds to simulation for $\mu=45 \mu \mathrm{m}^{-1}$ $\left(\mu=59 \mu \mathrm{m}^{-1}\right)$. (b) XAS for left (red) and right (blue) circular polarization and (c) XMCD for the $80 \mathrm{~nm}$-thick film at $60^{\circ}$ incidence, after the correction by $\alpha$. See text for more details. 
Therefore, in order to correct our data, it is sufficient to subtract the offset $\alpha$ from the measured $I^{\prime} / I_{0}^{\prime}$. The data in Fig. 10(a) are for the sum of left and right helicities. Therefore the offset to be subtracted from each individual helicity spectrum is $\alpha / 2$. Note that the offset subtraction is performed on the transmitted data, before the logarithm is taken, to obtain the absorption spectrum [see equation (1)]. The $\alpha$ contribution is likely present for all thicknesses, but only has a significant effect on the thickest samples. This is demonstrated by the simulations in Fig. 10(a), where the inclusion of $\alpha$ significantly changes the edge jump only for samples with thickness above $50 \mathrm{~nm}$.

The XAS and XMCD after the removal of $\alpha / 2$ from $I^{\mathrm{T}}$ are plotted in Figs. 10(b) and 10(c). The effect of the correction is clearest on the $L_{3}$ peak of the positive helicity spectrum and on the XMCD spectrum. The corresponding sum-rules results for this spectrum are plotted in Figs. 8(a) and $8(b)$ by the red open squares. The sum-rules results are in very good agreement with those from the thinner films, showing that the offset removal has satisfyingly corrected the data and, when properly considered, it can extend the thickness limitation on films measured using XEOL.

So far we have shown how to correct for the saturation effect. In the following we discuss the possible sources of this effect. As discussed in Section 3.7, we find that the XAS is qualitatively the same for all thicknesses for both flux conditions, ruling out a fixed offset in the detection system. Next we explored whether this effect could come from higher-order diffraction of the monochromator. Reducing the higherorder contamination of the beamline from $0.5 \%$ (standard setting) to $0.03 \%$ by changing the monochromator $c_{\mathrm{ff}}$ value (Piamonteze et al., 2012) did not result in a change in the $L_{3} / L_{2}$ branching ratio. Another possible factor that could cause the saturation effect is thickness inhomogeneity, as discussed by Stern \& Kim (1981) and also pointed out by Kallmayer et al. (2007). If surface roughness was to play a role in our case, and if it would be similar for all film thicknesses, then the offset should be larger for the thinner layers. We estimate that the offset for a roughness of $1 \mathrm{~nm}$, which is an upper bound from what we obtained from reflectivity measurements, would give an offset of the order of $1 \times 10^{-3}$ for a $10 \mathrm{~nm}$ film and $2 \times 10^{-6}$ for a $160 \mathrm{~nm}$ film. Comparing these values with the found value of $\alpha=1.4 \%$ it is clear that roughness alone cannot explain the saturation we observe. The only option which seems to explain our data is the contribution of fluorescence photons emitted from the Co film itself. Since the fluorescence photons have an energy below the $L_{3}$ absorption edge the attenuation length is about $1 / 2 \mu \mathrm{m}$ (Co pre-edge), and will be mostly transmitted to the substrate, where they create additional luminescence. The $L$ shell fluorescence efficiency is about $0.5 \%$ for Ni (Auerhammer et al., 1988) and it should be of the same order for Co. Only the fluorescence going in the direction of the substrate will generate luminescence, which approximately halves this value. These Co fluorescence photons will act as additional $\mathrm{X}$-ray intensity reaching the substrate generating additional optical luminescence. A rough estimate using the numbers discussed above suggests that the amount of fluorescence from a thick Co film reaching the substrate would be about $1.5-2 \%$ of $I_{0}$, which is of the same order as the correction factor used here.

The question that remains is: what is a safe thickness for XEOL measurements? In Fig. 10(a) we see that a significant divergence between the simulations for $\alpha=0$ and $\alpha=1.4 \%$ starts at around $30-50 \mathrm{~nm}$ effective thickness. Taking the absorption coefficient at the $L_{3}$ resonance from our simulation in Fig. 10(a) of $45 \mu \mathrm{m}^{-1}$ times an effective thickness of $50 \mathrm{~nm}$ we obtain $\mu t=2.25$, which is within the range suggested by previous works of $\mu t=2.6$ (Rose \& Shapiro, 1948) and 1.5 (Stern \& Kim, 1981) (for EXAFS). Guidelines for how to calculate $\mu$ at the resonance are given, for example, by Regan et al. (2001).

\section{Conclusions}

We have shown that XEOL detection from a substrate is a transmission equivalent detection mode of a thin film on top. We have measured the XEOL efficiency for a range of substrates, which should serve as a guideline for designing an XAS experiment based on XEOL detection. We have shown that XMCD sum rules applied to XEOL detected data give reasonable results independent of the analysis procedure. Analogously to transmission measurements, XEOL shows a saturation-like effect, which appears when part of the X-ray intensity is not absorbed by the sample. The saturation has a drastic effect on the result of the orbital sum rules. We have given guidelines for the film thickness to avoid this effect, keeping $\mu t<2.2$ at the maximum absorption, and we have shown how to successfully correct for the saturation when a range of film thicknesses is measured. In our case, we consider that the saturation effect comes from the X-ray fluorescence of the thin film itself which excites additional optical luminescence at the substrate.

Finally, XEOL is a good complementary detection method to TEY, due to the different probing depths allowing comparison between surface and bulk effects in thin films (Aeschlimann et al., 2018). Similarly to TFY it does not require a conductive sample. On the other hand, it does not suffer from known problems of TFY interpretation (de Groot, 2012; Liu et al., 2017). This method is useful not only in synchrotron beamlines but also in experiments using highharmonic-generation sources.

\section{Acknowledgements}

We wish to acknowledge E. Arenholz for helpful discussions about the XEOL setup at the Advanced Light Source. The measurements here were carried out using the EPFL/PSI endstation at the X-Treme beamline. We would like to thank the technical support of the X-Treme beamline in preparing the sample holders dedicated to XEOL measurements.

\section{Funding information}

Funding for this research was provided by: Schweizerischer Nationalfonds zur Förderung der Wissenschaftlichen 
Forschung (grant Nos. 169467 and 137657 to SRVA and YWW, respectively).

\section{References}

Achkar, A. J., Regier, T. Z., Wadati, H., Kim, Y.-J., Zhang, H. \& Hawthorn, D. G. (2011). Phys. Rev. B, 83, 081106.

Aeschlimann, R., Preziosi, D., Scheiderer, P., Sing, M., Valencia, S., Santamaria, J., Luo, C., Ryll, H., Radu, F., Claessen, R., Piamonteze, C. \& Bibes, M. (2018). Adv. Mater. 30, 1707489.

Auerhammer, J., Genz, H. \& Richter, A. (1988). Z. Phys. D - Atoms Mol. Clusters, 7, 301-307.

Bianconi, A., Jackson, D. \& Monahan, K. (1978). Phys. Rev. B, 17, 2021-2024.

Cao, Y., Shafer, P., Liu, X., Meyers, D., Kareev, M., Middey, S., Freeland, J. W., Arenholz, E. \& Chakhalian, J. (2015). Appl. Phys. Lett. 107, 112401.

Carra, P., Thole, B. T., Altarelli, M. \& Wang, X. (1993). Phys. Rev. Lett. 70, 694-697.

Elango, M. (1994). Radiat. Eff. Defects Solids, 128, 1-13.

Grabner, L. (1969). Phys. Rev. 177, 1315-1323.

Green, R. J., Regier, T. Z., Leedahl, B., McLeod, J. A., Xu, X. H., Chang, G. S., Kurmaev, E. Z. \& Moewes, A. (2015). Phys. Rev. Lett. 115, 167401.

Groot, F. M. F. de (2012). Nat. Chem. 4, 766-767.

Groot, F. M. F. de, Fuggle, J. C., Thole, B. T. \& Sawatzky, G. A. (1990). Phys. Rev. B, 41, 928-937.

Hanhan, S., Smith, A. M., Obst, M. \& Hitchcock, A. P. (2009). J. Electron Spectrosc. Relat. Phenom. 173, 44-49.

Iida, A. \& Noma, T. (1993). Jpn. J. Appl. Phys. 32, 28992902.

Kallmayer, M., Schneider, H., Jakob, G., Elmers, H. J., Balke, B. \& Cramm, S. (2007). J. Phys. D Appl. Phys. 40, 1552-1557.
Kjornrattanawanich, B., Korde, R., Boyer, C. N., Holland, G. E. \& Seely, J. F. (2006). IEEE Trans. Electron Devices, 53, 218-223.

Krause, M. O. \& Oliver, J. H. (1979). J. Phys. Chem. Ref. Data, 8 , 329-338.

Laan, G. van der (1999). J. Synchrotron Rad. 6, 694-695.

Liu, B., Piamonteze, C., Delgado-Jaime, M. U., Wang, R.-P., Heidler, J., Dreiser, J., Chopdekar, R., Nolting, F. \& de Groot, F. M. F. (2017). Phys. Rev. B, 96, 054446.

Meinert, M., Schmalhorst, J.-M., Reiss, G. \& Arenholz, E. (2011). J. Phys. D Appl. Phys. 44, 215003.

Nakajima, R., Stöhr, J. \& Idzerda, Y. U. (1999). Phys. Rev. B, 59, 6421-6429.

Piamonteze, C., Flechsig, U., Rusponi, S., Dreiser, J., Heidler, J., Schmidt, M., Wetter, R., Calvi, M., Schmidt, T., Pruchova, H., Krempasky, J., Quitmann, C., Brune, H. \& Nolting, F. (2012). J. Synchrotron Rad. 19, 661-674.

Regan, T., Ohldag, H., Stamm, C., Nolting, F., Lüning, J., Stöhr, J. \& White, R. (2001). Phys. Rev. B, 64, 214422.

Rose, M. E. \& Shapiro, M. M. (1948). Phys. Rev. 74, 1853-1864.

Stern, E. A. \& Kim, K. (1981). Phys. Rev. B, 23, 3781-3787.

Thole, B. T., Carra, P., Sette, F. \& van der Laan, G. (1992). Phys. Rev. Lett. 68, 1943-1946.

Tröger, L., Arvanitis, D., Baberschke, K., Michaelis, H., Grimm, U. \& Zschech, E. (1992). Phys. Rev. B, 46, 3283-3289.

Vaz, C. A. F., Moutafis, C., Buzzi, M. \& Raabe, J. (2013). J. Electron Spectrosc. Relat. Phenom. 189, 1-4.

Vaz, C. A. F., Moutafis, C., Quitmann, C. \& Raabe, J. (2012). Appl. Phys. Lett. 101, 083114.

Windsor, Y. W., Piamonteze, C., Ramakrishnan, M., Scaramucci, A., Rettig, L., Huever, J. A., Bothschafter, E. M., Bingham, N. S., Alberca, A., Avula, S. R. V., Noheda, B. \& Staub, U. (2017). Phys. Rev. B, 95, 224413.

Zubko, P., Gariglio, S., Gabay, M., Ghosez, P. \& Triscone, J.-M. (2011). Annu. Rev. Condens. Matter Phys. 2, 141-165. 